\title{
Uniqueness Properties of Hardy Space Functions出
}

\author{
Steven G. Krantz
}

November 8, 2018

\begin{abstract}
We study boundary uniqueness properties of Hardy space functions in several complex variables. Along the way, we develop properties of the Lumer Hardy space.
\end{abstract}

\section{Introduction}

Ever since the early work of Riesz and Hardy, the Hardy spaces have been an important cornerstone of the harmonic analysis of complex variable theory. Even so, the theory of Hardy spaces in several complex variables is not nearly as well developed as one would like.

In particular, many of the basic results about boundary uniqueness for these function spaces have not been rigorously and completely established (although in some instances they are a part of the folklore). It is our purpose in this paper to set some of these matters straight.

Throughout this paper, if $\Omega \subseteq \mathbb{C}^{n}$ is a given bounded domain with $C^{2}$ boundary, then we let $d \sigma$ denote the usual boundary area measure on $\partial \Omega$. In other words, $d \sigma$ is $(2 n-1)$-dimensional Hausdorff measure. (See [KRA1, Appendix II].) In situations when we are dealing with a domain $\Omega_{j}$ or $\Omega_{\epsilon}$, we shall denote the boundary measure by $d \sigma_{j}$ or $d \sigma_{\epsilon}$.

\footnotetext{
${ }^{1}$ Subject Classification Numbers: 32A35, 32A50, 32A70, 30H10.

${ }^{2}$ Key Words: Hardy space, boundary behavior, boundary uniqueness, Lumer Hardy space.
} 
A basic fact of life is that a Hardy space function $f$ on a smoothly bounded domain $\Omega$ in $\mathbb{C}^{n}$ has nontangential boundary limits (indeed admissible boundary limits - see [STE1] and [KRA1, Ch. 8]) almost everywhere with respect to the usual boundary area measure $d \sigma$. Thus we may analyze the boundary trace function $\widetilde{f}$ and pose questions about it. For example, if the boundary trace function $\widetilde{f}$ vanishes on a set of positive $\sigma$ measure, does it then follow that $f \equiv 0$ ? Such a result is well known in one complex variable, and follows from the canonical factorization (in particular, from properties of the Nevanlinna class - see below). But it is not generally established in several complex variables.

Likewise, a biholomorphic mapping $\Phi: \Omega_{1} \rightarrow \Omega_{2}$ of smoothly bounded domains will have a boundary trace $\widetilde{\Phi}$. It follows either from the properness of the mapping (and its inverse) or from the maximum principle that $\widetilde{\Phi}$ maps $\partial \Omega_{1}$ to $\partial \Omega_{2}$. And of course $\widetilde{\Phi^{-1}}$ enjoys a similar property.

It is well known, in the one variable context, that $\widetilde{\Phi}$ takes sets of zero boundary measure to sets of zero boundary measure and sets of positive boundary measure to sets of positive boundary measure (this follows, for instance, because of Painlevés theorem ${ }^{3}$ that the biholomorphic mapping continues smoothly to the boundary, and so does its inverse). Is an analogous result true in several complex variables (in which context we do not have a general boundary smoothness theorem)?

Interestingly, some of these questions relate to properties of Lumer's Hardy spaces. In the course of answering the above-raised questions, we also develop some relevant properties of Lumer's spaces. We also use Lumer's Hardy spaces in some of the proofs.

It may be noted that the answers to some of these queries are straightforward if it is known that biholomorphic mappings continue smoothly and univalently to the boundary. So, for example, we may affirmatively answer the second question for strongly pseudoconvex domains and finite type domains (see [KRA1]). But our purpose here is to come up with statements and proofs that are valid for all smoothly bounded domains in $\mathbb{C}^{n}$.

It is a pleasure to thank E. Bedford, S. R. Bell, R. Burckel, and J. A. Cima for helpful remarks regarding the work in this paper.

\footnotetext{
${ }^{3}$ See $[\mathrm{BUR}]$ for the provenance of the Painelvé result.
} 


\section{Basic Concepts and Notation}

In all of our discussions, a domain in $\mathbb{C}^{n}$ will be a connected, open set. We will let $\Omega$ denote a bounded domain in $\mathbb{C}^{n}$ with $C^{2}$ boundary. So

$$
\Omega=\left\{z \in \mathbb{C}^{n}: \rho(z)<0\right\},
$$

with $\rho$ a given $C^{2}$ defining function satisfying $\nabla \rho \neq 0$ on $\partial \Omega$.

Recall, for $0<p<\infty$, that

$H^{p}(\Omega)=\left\{f\right.$ holomorphic on $\left.\Omega: \sup _{\epsilon>0} \int_{\rho(\zeta)=-\epsilon}|f(\zeta)|^{p} d \sigma_{\epsilon}(\zeta)^{1 / p} \equiv\|f\|_{H^{p}(\Omega)}<\infty\right\}$,

where $d \sigma_{\epsilon}$ is boundary area measure on the boundary of $\Omega_{\epsilon} \equiv\{z: \rho(z)<$ $-\epsilon\}$. It is known (see [KRA1, Ch. 8]) that this definition is independent of the choice of defining function $\rho$. We also define $H^{\infty}(\Omega)$ to be the bounded holomorphic functions on $\Omega$ with the obvious supremum norm.

We say that a function $g$ on a domain $\Omega$ has a harmonic majorant (resp. pluriharmonic majorant) $u$ if the harmonic (resp. pluriharmonic) function $u$ satisfies $|g(z)| \leq u(z)$ for all $z \in \Omega$. It is a basic fact that a holomorphic function $f$ on $\Omega$ lies in $H^{p}(\Omega), 0<p<\infty$, if and only if $|f|^{p}$ has a harmonic majorant (see [STE1], [KRA1, Ch. 8]).

The trouble with $H^{p}$ as we have defined and discussed it here is that it is not obviously a biholomorphically invariant notion (as is, for instance, the concept of Bergman space see [KRA1, Ch. 1]). For this reason it is useful to have the concept of Lumer Hardy space. We say that a holomorphic function $f$ on $\Omega$ is in the Lumer Hardy p-space $\mathcal{L} H^{p}$ if the function $|f|^{p}$ has a pluriharmonic majorant. Since pluriharmonic functions are preserved by biholomorphic mappings of several complex variables, it is clear that the concept of Lumer Hardy space is a biholomorphic invariant.

The norm on $\mathcal{L} H^{p}$ is specified as follows. Fix a point $P_{0} \in \Omega$. Then $\|f\|_{\mathcal{L H}^{p}} \equiv \inf u^{1 / p}\left(P_{0}\right)$, where the infimum is taken over all possible pluriharmonic majorants $u$ of $|f|^{p}$. Note that different choices of $P_{0}$ give equivalent norms.

In spite of its favorable property of invariance, the Lumer space has a number of pathological properties. For instance (see [RUD]), the Lumer space $\mathcal{L} H^{2}$ is a Banach space but not a Hilbert space.

It is clear, just because a pluriharmonic function is obviously harmonic, that $\mathcal{L} H^{p}$ is a subspace of $H^{p}$.

Indeed, we have 
Proposition 2.1 The space $\mathcal{L} H^{2}(\Omega)$ in $\mathbb{C}^{n}, n \geq 2$, is a Banach subspace of $H^{2}(\Omega)$ but is not a Hilbert space.

Proof: For simplicity take $\Omega$ to be the unit ball $B$. Rudin [RUD] shows that $\mathcal{L} H^{2}$ contains a closed subspace that is isomorphic to $\ell^{\infty}$. Since every closed subspace of a Hilbert space is a Hilbert space, it then follows that $\mathcal{L} H^{2}$ is not a Hilbert space.

To see that $\mathcal{L} H^{2}$ is a Banach space, suppose that $g_{j}$ is a Cauchy sequence in $\mathcal{L} H^{2}$. Then each $\left|g_{j}\right|^{2}$ has a least pluriharmonic majorant $u_{j}$ and $\left\{u_{j}\left(P_{0}\right)\right\}$ is Cauchy. But the Harnack inequalities, together with a connectedness argument, then show that the $u_{j}$ have a Cauchy subsequence $\left\{u_{j_{k}}\right\}$ that converges uniformly on compact sets. It follows that the $g_{j_{k}}$ converge. So the space is complete, and hence is a Banach space.

Proposition 2.2 The space $\mathcal{L} H^{p}$ is a proper subspace of $H^{p}$.

Proof: See $[\mathrm{LUM}]$ for a version of this result.

We restrict attention to $\Omega=B$, the unit ball in $\mathbb{C}^{2}$, and to $p=2$. Set

$$
f(z)=\left(1-z_{1}\right)^{-1}
$$

Then, because

$$
\left|z_{2}\right|^{2}<1-\left|z_{1}\right|^{2} \leq 2\left|1-z_{1}\right|
$$

it is easy to see that $f \in H^{2}(B)$. But it is clear that $|f|^{2}$ does not have a harmonic majorant on the complex line $\{\zeta\langle 1,0, \ldots, 0\rangle: \zeta \in \mathbb{C}\}$ and hence does not have a pluriharmonic majorant on $B$.

Proposition 2.3 The dual of $\mathcal{L} H^{2}(B)$ does not equal the dual of $H^{2}(B)$.

Proof: Of course this follows just by functional analysis from Proposition 2.2. But it is worthwhile to see the result explicitly.

We restrict attention to the unit ball $B$ in complex dimension $n=2$. If $g$ is holomorphic on the unit ball in $\mathbb{C}^{2}$, then

$$
g(z)=\sum_{j} g_{j}(z)
$$


where each $g_{j}$ is a homogeneous polynomial of degree $j$. Rudin [RUD] proved the following: If $P \in \partial B$ and $j \geq 0$ is fixed, then

$$
\left|g_{j}(P)\right| \leq\|g\|_{\mathcal{L} H^{2}}
$$

With this result in hand, we let $P=(1,0)$ and consider the linear functionals on $\mathcal{L} H^{2}$ defined by

$$
\varphi_{n}(f)=\frac{\partial^{n}}{\partial z_{1}^{n}} f(P) \quad, \quad n \in\{1,2, \ldots\} .
$$

This is a bounded linear functional by (2.3.1).

We apply the functional $\varphi_{n}$ to the $H^{2}$ function given by

$$
f^{k}\left(z_{1}, z_{2}\right)=\frac{\left(z_{1}+1\right)^{k}}{2^{k}}
$$

for $k \in\{1,2, \ldots\}$. Of course this is a holomorphic peaking function at the boundary point $P=(1,0) \in \partial B$.

Obviously $\varphi_{n}\left(f^{k}\right)=k(k-1) \cdots(k-(n-1)) \cdot 2^{k-n} / 2^{k}=k(k-1) \cdots(k-$ $(n-1)) / 2^{n}$. On the other hand, the value of the least harmonic majorant of $\left|\left(f^{k}\right)^{2}\right|$ (which is simply the solution of the Dirichlet problem with $\left.\left|\left(f^{k}\right)\right|^{2}\right|_{\partial B}$ as boundary data) at 0 is $\leq C \cdot(1+\alpha)^{2 k} / 2^{2 k}$ for some $0<\alpha<\sqrt{2}-1$. Here we have simply used the mean value property for harmonic functions (or else we can see this assertion by way of harmonic measure). So the corresponding $H^{2}$ norm is $\leq C \cdot(1+\alpha)^{k} / 2^{k}$. And, if $C \cdot(1+\alpha)^{k} / 2^{k}$ bounds $k(k-1) \cdots(k-(n-1)) / 2^{n}$ uniformly in $n$, then

$$
\frac{k(k-1) \cdots(k-(n-1))}{2^{n}} \leq C \cdot \frac{(1+\alpha)^{k}}{2^{k}}
$$

hence

$$
k(k-1) \cdots(k-(n-1)) \leq C \cdot(1+\alpha)^{k} \cdot 2^{n-k} .
$$

We take $n=k / 2$ (assuming that $k$ is even and [k/2] otherwise) and obtain

$$
k(k-1) \cdots(k-((k / 2)-1))<C .
$$

This is clearly a contradiction if $k$ is large. So $\varphi_{n}$ is not bounded on $H^{2}$.

It appears to be rather difficult to give a complete intrinsic description of the dual of $\mathcal{L} H^{2}$. 


\section{Boundary Regularity}

A first natural question to ask is whether a biholomorphic mapping of domains extends to an invertible mapping of the boundaries.

In one complex variable this question is fairly easy. For suppose that $\phi: \Omega_{1} \rightarrow \Omega_{2}$ is a conformal mapping of domains in $\mathbb{C}$, each domain having a Jordan curve as boundary. Then a classical theorem of Carathéodory [GRK] tells us that $\phi$ continues to a bicontinuous mapping of the closures. The univalence follows easily.

In several complex variables we know, for domains with $C^{2}$ boundary let us say, that a biholomorphic mapping $\Phi: \Omega_{1} \rightarrow \Omega_{2}$ has an almost-everywhere defined boundary mapping $\widetilde{\Phi}$ and we also know that the inverse mapping $\Phi^{-1}$ has an almost-everywhere defined boundary mapping $\widetilde{\Phi^{-1}}$. But we do not know that $\widetilde{\Phi^{-1}} \circ \widetilde{\Phi}=$ id or that $\widetilde{\Phi} \circ \widetilde{\Phi^{-1}}=\mathrm{id}$. The next result addresses this matter.

Even in one variable it appears that this matter is not well documented. So we treat that case first.

Theorem 3.1 Let $\Omega_{1}, \Omega_{2}$ be bounded domains in $\mathbb{C}$ with $C^{2}$ boundary. Let $\Phi: \Omega_{1} \rightarrow \Omega_{2}$ be a conformal mapping. Then the boundary mappings $\widetilde{\Phi}$ and $\widetilde{\Phi^{-1}}$ are inverse to each other.

Proof: I thank S. R. Bell for helpful remarks about this proof.

First it must be noted that $\Phi$ and $\Phi^{-1}$ each extend to be $C^{1}$ on the closures (see, for instance, $[\mathrm{BEK}],[\mathrm{POM}]$ ). So we may think of $\Phi: \overline{\Omega_{1}} \rightarrow \overline{\Omega_{2}}$ as a diffeomorphism and $\Phi^{-1}: \overline{\Omega_{2}} \rightarrow \overline{\Omega_{1}}$ as a diffeomorphism. It follows from the maximum principle, or from the properness of the mappings, that $\Phi$ takes $\partial \Omega_{1}$ to $\partial \Omega_{2}$ and $\Phi^{-1}$ takes $\partial \Omega_{2}$ to $\partial \Omega_{1}$.

It follows from the Hopf lemma (see [KRA2]) that $\nabla \Phi$ is nonvanishing on $\partial \Omega_{1}$. The nonvanishing derivative together with the smooth extension shows that each boundary curve of $\Omega_{1}$ gets mapped to a particular boundary curve of $\Omega_{2}$. And we see that, as a domain point $p$ traverses a boundary curve in the domain $\partial \Omega_{1}$, the corresponding image point $\Phi(p)$ in $\partial \Omega_{2}$ traverses a boundary curve a certain number of times. But the argument principle tells us that that number of times is one. That proves the result.

Alternative View of Theorem 3.1: In case the boundaries of the domains in question are not smooth, a result is still possible. Consider for instance 
the case when $\partial \Omega_{1}, \partial \Omega_{2}$ have Lipschitz boundary. [We make this particular geometric hypothesis so that each boundary point has a well defined concept of nontangential convergence, and also of radial convergence.] Since each boundary curve (or curves) is Jordan, Carathéodory's theorem implies that the conformal mapping extends continuously to the boundary, and so does its inverse.

Assume that $P \in \partial \Omega_{1}$ is a boundary point at which $\Phi$ has a radial limit. If $\nu_{P}$ is the unit outward normal vector at $P$, then we may consider $\lim _{t \rightarrow 0^{+}} \Phi\left(P-t \nu_{P}\right)$. This limit will certainly exist (this is what we think of as the "radial limit"), and $t \mapsto \Phi\left(P-t \nu_{P}\right), 0<t<\epsilon_{0}$, will describe some curve in $\Omega_{2}$. And the fact that $\Phi^{-1} \circ \Phi=\mathrm{id}$ implies that $\lim _{t \rightarrow 0^{+}} \Phi^{-1} \circ \Phi\left(P-t \nu_{P}\right)$ exists. But the generalized Lindelöf principle proved in [LEV] then implies that the nontangential limit of $\Phi^{-1}$ at $\widetilde{\Phi}(P)$ exists. From this we may conclude that the limit that defines $\widetilde{\Phi^{-1}}$ coincides with the pointwise boundary limit of $\Phi$. Therefore $\widetilde{\Phi^{-1} \circ \Phi}=\widetilde{\Phi^{-1}} \circ \widetilde{\Phi}=\widetilde{\mathrm{id}}=\mathrm{id}$. And that is what we wish to conclude.

We note for the record that neither version of Theorem 3.1 obtains (at least not immediately) in the context of several complex variables. For there certainly is no theorem, except in special cases, that says that biholomorphic mappings extend continuously to the boundary (or smoothly to the boundary). And the Lindelöf principle in several variables is different from that in one complex variable (see [CIK], [KRA3]).

Now we turn to the several variable situation. Let $\Omega_{1}, \Omega_{2}$ be bounded domains in $\mathbb{C}^{n}$ with $C^{2}$ boundary. Let $\Phi: \Omega_{1} \rightarrow \Omega_{2}$ be a biholomorphic mapping. Then there is a $\sigma_{1}$-almost everywhere defined boundary mapping $\widetilde{\Phi}: \partial \Omega_{1} \rightarrow \partial \Omega_{2}$ and there is a $\sigma_{2}$-almost everywhere defined boundary mapping $\widetilde{\Phi^{-1}}: \partial \Omega_{2} \rightarrow \partial \Omega_{1}$. The mapping $\Phi$ converges to $\widetilde{\Phi}$ both nontangentially and admissibly. Likewise for the mappings $\Phi^{-1}$ and $\widetilde{\Phi^{-1}}$.

The assertions about the existence of $\widetilde{\Phi}$ and $\widetilde{\Phi^{-1}}$, and about the convergence of $\Phi$ to $\widetilde{\Phi}$ and the convergence of $\Phi^{-1}$ to $\widetilde{\Phi^{-1}}$ follow from standard results about the boundary behavior of $H^{\infty}$ functions. See [STE1] and [KRA1, Ch. 8].

Theorem 3.2 The mapping $\widetilde{\Phi}$ and the mapping $\widetilde{\Phi^{-1}}$ are each one-to-one (almost everywhere) and onto (almost everywhere). 
First Proof of Theorem 3.2: We need to show that $\widetilde{\Phi}$ is both one-toone and onto in a suitable measure-theoretic sense. Let us begin with the surjectivity. Seeking a contradiction, we suppose that the image of $\widetilde{\Phi}$ misses a set $H \subseteq \partial \Omega_{2}$ of positive $\sigma_{2}$ measure. Let $f$ be the characteristic function of $H$. For $\psi \in \mathcal{L} H^{2}\left(\Omega_{1}\right)$, we consider the linear functional

$$
\lambda: \psi \mapsto \int_{\partial \Omega_{1}}(f \circ \widetilde{\Phi})(\zeta) \cdot \psi(\zeta) d \sigma_{1}(\zeta)
$$

Then it is immediate that $\lambda$ is the zero functional. But $f$ certainly does not induce the zero functional on $\mathcal{L} H^{2}\left(\Omega_{2}\right)$. And a biholomorphic mapping of domains will of course induce an isomorphism of $\mathcal{L} H^{2}\left(\Omega_{1}\right)^{*}$ with $\mathcal{L} H^{2}\left(\Omega_{2}\right)^{*}$. So that is a contradiction. Thus $\widetilde{\Phi}$ is onto.

Next we treat the univalence. This point is tricky because we must determine how to formulate this univalence in an almost-everywhere sense. Suppose that $f_{1}$ and $f_{2}$ are continuous functions with disjoint compact supports on $\partial \Omega_{1}$ which each map to the same function $g$ on $\partial \Omega_{2}$ in the sense that $g \circ \widetilde{\Phi}=f_{1}$ on the domain of $f_{1}$ and $g \circ \widetilde{\Phi}=f_{2}$ on the domain of $f_{2}$. Consider the functionals

$$
\lambda_{j}(\varphi)=\int_{\partial \Omega_{1}} \varphi \circ \widetilde{\Phi}(\zeta) \cdot f_{j}(\zeta) d \sigma_{1}(\zeta)
$$

on $\mathcal{L} H^{2}\left(\Omega_{2}\right), j=1,2$. Now $\lambda_{j}$ may be thought of as the push-forward of the functional on $\mathcal{L} H^{2}\left(\Omega_{1}\right)$ induced by $f_{j}$. On the other hand, the HahnBanach theorem tells us that the functional $\lambda_{j}$ extends to a linear functional on $L^{2}\left(\partial \Omega_{2}\right)$ and is therefore given by inner product with an $L^{2}\left(\partial \Omega_{2}\right)$ function $g_{j}$. And $g_{j}$ is supported on the image of the support of $f_{j}$ under $\widetilde{\Phi}$. In fact $g_{j}$ must be $g$.

But this says that $\lambda_{1}=\lambda_{2}$. And that tells us that the induced map from $\mathcal{L} H^{2}\left(\Omega_{1}\right)^{*}$ to $\mathcal{L} H^{2}\left(\Omega_{2}\right)^{*}$ is not univalent. That is false.

This contradiction completes the proof of univalence.

Second Proof of Theorem 3.2: Here we prove a weak version of the univalence. Restrict attention to $\mathbb{C}^{2}$. Assume that $\Omega_{1} \subseteq \mathbb{C}^{2}$ and $\Omega_{2} \subseteq \mathbb{C}^{2}$. Suppose that $E \subseteq \partial \Omega_{1}$ has positive $\sigma_{1}$ measure and that $E$ is mapped by $\widetilde{\Phi}$ to a constant $\alpha \in \partial \Omega_{2}$. We will derive therefrom a contradiction.

Let $P \in E$ be a point of $\sigma_{1}$ density. Using Fubini's theorem, find a complex line $\ell$ transversal to $\partial \Omega_{1}$ so that $\ell \cap \partial \Omega_{1}$ near $P$ is a one-dimensional, 
smooth real curve and $\ell \cap E$ is a subset of that curve having positive linear measure. We may conclude that $\widetilde{\Phi}$ maps $\ell \cap E$ to $\alpha$ (see [NAR]). But then we can look at a component $\widetilde{\Phi}_{j}$ of $\widetilde{\Phi}, j=1,2$; it clearly maps $\ell \cap E$ to $\alpha_{j}$. So we have a bounded, scalar-valued holomorphic function of one complex variable that is constant on a piece of the boundary having positive measure. So $\left.\widetilde{\Phi}_{j}\right|_{\ell \cap \Omega_{1}}$ is constant. Since we can make this argument for uncountably many distinct complex lines $\ell$, we conclude that $\widetilde{\Phi}_{j}$ is constant for each $j=1,2$. Thus $\Phi$ is constant. That is of course impossible. We conclude that $\widetilde{\Phi}$ is set-theoretically one-to-one (in a weak sense).

Now we wish to show that $\widetilde{\Phi}$ is set-theoretically onto. Seeking a contradiction, we suppose that the image of $\widetilde{\Phi}$ misses a set $F \subseteq \partial \Omega_{2}$ of positive $\sigma_{2}$ measure. We can then, as above, find a transversal complex line $\ell$ so that $\ell \cap \partial \Omega_{2}$ is a one-dimensional, smooth real curve and $\ell \cap F$ is a subset of that curve having positive linear measure.

But then we see that $\widetilde{\Phi}^{-1}$ maps $\ell \cap F$ to the empty set. That clearly contradicts our argument in the first two paragraphs of this proof.

It should be noted that we have proved that $\widetilde{\Phi}$ is both univalent and surjective (in a certain measure-theoretic sense). Of course the same result

holds true for $\widetilde{\Phi^{-1}}$. But we have not proved that $(\widetilde{\Phi})^{-1}=\widetilde{\Phi^{-1}}$ nor that $\widetilde{\Phi^{-1}} \circ \widetilde{\Phi}=$ id.

\section{The First Main Result}

The theorem that we treat in this section is as follows. Let $\Omega_{1}, \Omega_{2}$ be bounded domains in $\mathbb{C}^{n}$ with $C^{2}$ boundary. Let $d \sigma_{j}$ be the usual area measure on $\partial \Omega_{j}$, $j=1,2$. Let $\Phi: \Omega_{1} \rightarrow \Omega_{2}$ be biholomorphic. Then $\Phi$ has boundary trace $\widetilde{\Phi}$ and $\Phi^{-1}$ has boundary trace $\widetilde{\Phi^{-1}}$.

Theorem 4.1 Let $E \subseteq \partial \Omega_{1}$ have $\sigma_{1}$ measure 0 and $F \subseteq \partial \Omega_{1}$ have positive $\sigma_{1}$ measure. Then $\widetilde{\Phi}(E)$ has $\sigma_{2}$ measure 0 and $\widetilde{\Phi}(F)$ has positive $\sigma_{2}$ measure.

First Proof of Theorem 4.1: The first of these statements is the contrapositive of the second. So we concentrate on proving the second.

Seeking a contradiction, we supposed that $F \subseteq \Omega_{1}$ is a set of positive $\sigma_{1}$ measure and that $G \equiv \widetilde{\Phi}(F)$ has zero $\sigma_{2}$ measure. Define $X$ to be the collection of those functions which are characteristic functions of measurable sets 
in $\partial \Omega_{2}$ that are disjoint from $G$. And let $Y$ be the linear space generated by $\mathcal{L} H^{2}\left(\Omega_{2}\right) \cup X$. [Here we identify each element of $\mathcal{L} H^{2}\left(\Omega_{2}\right)$ with its boundary function.]

Consider the linear functional on $\mathcal{L} H^{2}\left(\Omega_{2}\right)$ defined by

$$
\phi(f)=\int_{\partial \Omega_{1}} \chi_{F}(\zeta) \cdot(f \circ \Phi)(\zeta) d \sigma_{1}(\zeta) .
$$

We may extend this functional to $X$ and then to $Y$ by simply setting $\phi(g)=0$ for any $g \in X$ and then extending by linearity. Notice that this extended functional is also specified by the integral in (4.1.1).

Now we may apply the Hahn-Banach theorem to extend the functional $\phi$ to a new functional $\widetilde{\phi}$ on all of $L^{2}\left(\partial \Omega_{2}\right)$. So of course $\widetilde{\phi}$ is given by integration against an $L^{2}\left(\partial \Omega_{2}\right)$ function $p(\zeta)$. And, since $\phi$ (and hence $\widetilde{\phi}$ ) annihilates $X$, it must be that $p$ lives on $G$. But we know that $G$ has $\sigma_{2}$ measure 0 . So we are forced to conclude that the extended functional $\widetilde{\phi}$ is the identically zero functional. But the functional

$$
\psi(h)=\int_{\partial \Omega_{1}} \chi_{F}(\zeta) \cdot h(\zeta) d \sigma_{1}(\zeta)
$$

is certainly not the zero functional (simply take $h$ to be a polynomial with positive real part).

So we have determined that the canonical mapping induced by $\widetilde{\Phi}$ from the dual space of $\mathcal{L} H^{2}\left(\Omega_{1}\right)$ to the dual space of $\mathcal{L} H^{2}\left(\Omega_{2}\right)$ sends a nonzero functional to the zero functional. And that is impossible.

Second Proof of Theorem 4.1 For convenience let us work in $\mathbb{C}^{2}$. Let $E \subseteq \partial \Omega_{1}$ have positive $\sigma_{1}$ measure. We claim that $\widetilde{\Phi}(E)$ cannot have $\sigma_{2}$ measure zero. Seeking a contradiction, suppose not.

Let $P \in E$ be a point of $\sigma_{1}$ density. Choose a complex line $\ell$ which is transversal to the boundary and so that $\ell \cap \partial \Omega_{1}$ is a smooth curve and further so that $\ell \cap E$ is a subset of that curve having positive linear measure. It is easy to see, using Fubini's theorem simultaneously in the domain and the range, that this $\ell$ and the corresponding curve may be chosen so that the image of $\ell \cap E$ under $\widetilde{\Phi}$ has measure 0 . Then $\left.\Phi\right|_{\ell \cap \Omega_{1}}$ must be identically 0 by the one-variable theory. Since we can choose uncountably many distinct complex lines $\ell$ of this nature, we may conclude that $\Phi$ must be indentically 0 . That is a contradiction. 
As usual, the statement that sets of measure 0 cannot get mapped to sets of positive measure is just the contrapositive of what we have just proved.

It would be incorrect to infer that a set of zero $\sigma$ measure in the boundary of a domain cannot be a set of determinacy. For instance, if a Hardy space function on the bidisc vanishes on a set of positive 2-dimensional measure in the distinguished boundary of the polydisc, then that function is identically zero. See Theorem 5.1 below.

Example 4.2 The result of the theorem is false if the mapping is not biholomorphic. As a simple example, let us first look at the one-complex-variable situation. Let $D \subseteq \mathbb{C}$ be the unit disc. Recall the notable theorem of Bagemihl and Seidel [BAS]:

Theorem 4.3 Let $E \subseteq \partial D$ be an $F_{\sigma}$ of first category. Let $\varphi$ be any continuous function on $D$. Then there is a holomorphic function $f$ on $D$ so that

$$
\lim _{r \rightarrow 1^{-}}(\varphi(r \xi)-f(r \xi))=0
$$

for every $\xi \in E$.

Now let $E \subseteq \partial D$ be an $F_{\sigma}$ of first category and full measure. Let $F \subseteq E$ have boundary measure 1 (note that $\partial D$ has boundary measure $2 \pi$ ). Certainly there exists a continuous, complex-valued function $\varphi$ on $D$ with values in the disc, finite unimodular radial boundary limit at every point of $E$ (call the boundary function $\widetilde{\varphi}$ ) and so that $\widetilde{\varphi}(F)$ has measure 0 . Now the theorem of Bagemihl and Seidel guarantees the existence of a holomorphic function $f$ on $D$ which has the same radial limits as $\varphi$ at each point of $E$. In particular, the boundary function $\widetilde{f}$ maps $F$ to a set of boundary measure 0 .

In the several complex variable context, consider $D^{2}=D \times D$ and define $\mathcal{F}\left(z_{1}, z_{2}\right)=f\left(z_{1}\right)$ with $f$ as in the preceding paragraph. Then this $\mathcal{F}$ takes the set $F \times D$ in the boundary of $D^{2}$ to a set of measure zero.

Note that the paper [HAS] contains a version of the Bagemihl-Seidel theorem in several complex variables that is valid on convex domains. In particular, it is valid on the unit ball in $\mathbb{C}^{n}$. They state their result for holomorphic functions, but it is true as well for holomorphic mappings. Thus, as in the first paragraph, one can generate an example directly on the unit ball of $\mathbb{C}^{n}$. 
It should be noted that, in general, the functions provided by the BagemihlSeidel theorem in $[\mathrm{BAS}]$ or in $[\mathrm{HAS}]$ are not bounded, and not necessarily in any $H^{p}$ class (nor in the Nevanlinna class). So, strictly speaking, the examples described in the three paragraphs following Theorem 4.3 are not counterexamples to Theorem 4.1 in the case that $\Phi$ is not biholomorphic.

\section{Boundary Uniqueness}

First we recall the result in one complex variable.

Theorem 5.1 Let $f$ be an $H^{p}$ function on the unit disc $D, 0<p<\infty$. Let $\widetilde{f}$ be the boundary trace of $f$. If $\widetilde{f}(\zeta)=0$ for $\zeta$ in a set $E$ of positive linear measure in $\partial D$, then $f \equiv 0$.

Proof: Seeking a contradiction, we suppose that $f$ is not identically equal to 0 .

Certainly $f$ is in the Nevanlinna class $N$. Therefore (see [DUR]), $g \equiv 1 / f$ also lies in $N$. And obviously $\widetilde{g}=\infty$ on $E$. But, setting $g_{r}\left(e^{i \theta}\right)=g\left(r e^{i \theta}\right)$, we know that $\log ^{+}\left|g_{r}\right| \rightarrow \log ^{+}|\widetilde{g}|$ as $r \rightarrow 1^{-}$, and that $\log ^{+}|\widetilde{g}|$ is integrable. On the other hand, $\log ^{+}\left|g_{r}\right| \rightarrow \infty$ on the set $E$. That is a contradiction.

See [LEM, pp. 3, 17] for related results.

Theorem 5.2 Let $f$ be a Hardy space function on the smoothly bounded domain $\Omega \subseteq \mathbb{C}^{n}$, and suppose that the boundary trace function $\widetilde{f}$ vanishes on a set $E \subseteq \partial \Omega$ of positive $\sigma$ measure. Then $f \equiv 0$ on $\Omega$.

Proof: For simplicity of notation we take the complex dimension of the ambient space to be 2 .

By a simple change of coordinates, we may suppose that $P \in \partial \Omega$ is a point of $\sigma$-measure density of $E$ and that the unit outward normal direction to the boundary at $P$ is $\mathbf{1}=\langle 1,0\rangle$. We may also suppose that $P$ is the origin of coordinates.

Now let $\mathbf{d}$ be the complex tangential disc to $\partial \Omega$ at $P$ given by

$$
\zeta \longmapsto(1, \zeta) \equiv Z
$$

for $\zeta$ small (say $|\zeta|<\eta$ ). For each such point $(1, \zeta)=Z$, we set

$$
D_{Z}=\Omega \cap\{Z+\xi 1: \xi \in \mathbb{C},|\xi|<\eta\} .
$$


Then each $D_{Z}$ is a complex disc that is transverse to the boundary $\partial \Omega$ and the collection of all $D_{Z}$ foliates a boundary neighborhood of $P \in \partial \Omega$. Let $e_{Z}=D_{Z} \cap \partial \Omega$ for each $Z$.

We know that $f$ has (let us say) nontangential boundary limit 0 at each point of $E$. Hence, by Fubini's theorem, there are an uncountable collection of $Z$ so that $\left.f\right|_{D_{Z}}$ has nontangential boundary limit 0 on a subset of $e_{Z}$ that has positive linear measure.

Passing to a possibly smaller uncountable set of $Z$, we can again apply Fubini's theorem to say that $f$ restricted to each $D_{Z}$ is in $H^{p}$ of that $D_{Z}$. More precisely, let $\epsilon_{1}>\epsilon_{2}>\cdots \rightarrow 0$. Using our earlier notation from Section 2 , and invoking the fact that $f \in H^{p}(\Omega)$, we know that $|f|^{p}$ is integrable on each $\partial \Omega_{\epsilon_{j}} \cap D_{Z}$. Now using standard results about equivalence of different $H^{p}$ norms (see [KRA1, Ch. 8]), we may conclude that $|f|^{p}$ is integrable on level sets of $\partial D_{Z}$, each $Z$. So $f \in H^{p}\left(D_{Z}\right)$, each $Z$. Hence we may apply Theorem 5.1 on each $D_{Z}$ to conclude that $\left.f\right|_{D_{Z}}=0$, each $Z$.

But the structure of the zero set of a holomorphic function on a domain in $\mathbb{C}^{2}$ is well known (see [KRA1, Ch. 7]. It is not possible for a nonzero holomorphic function to vanish on uncountably many disjoint leaves. We can only conclude that $f \equiv 0$.

One of the more interesting results of classical function theory is the following.

Proposition 5.3 Let $f$ be a holomorphic function on the unit disc $D$ which has nontangential boundary limit 0 on a set $E \subseteq \partial D$ of positive measure. Then $f \equiv 0$.

Remark 5.4 Note that, in this result, we are not assuming that $f \in H^{p}$ for some $p$. In fact there is no growth condition on $f$. But we are looking at nontangential limits instead of radial limits. This is a result of Privalov, for which see [COL, pp. 145-146].

Proof of the Proposition: By a standard measure-theoretic argument (see [STE2]), we may take the nontangential approach regions to all have the same aperture. Let $U$ be the domain obtained by taking the union of all the nontangential approach regions which terminate at points of $E$, together with a suitable simply connected, relatively compact subset of $D$. Then $U$ is a simply connected domain with Lipschitz boundary. So there is a conformal 
mapping $\varphi$ of $U$ to the unit disc $D$. But then $f \circ \varphi^{-1}$ is a holomorphic function on $D$ that extends continuously to $\partial D$ and vanishes on a boundary set of positive measure.

But now we are dealing with a bounded holomorphic function. So of course it must be that $f \circ \varphi^{-1} \equiv 0$ and so $f \equiv 0$.

What is remarkable and notable is that the proposition is false if "nontangential boundary limit" is replaced by "radial boundary limit." This follows immediately from the classical theorem of Bagemihl and Seidel [BAS] cited above.

Now we have a result of a similar nature in several complex variables. For comparison purposes, it is worth mentioning the theorem of Hakim and Sibony [HAS].

Theorem 5.5 Let $\Omega \subseteq \mathbb{C}^{n}$ be a smoothly bounded domain. Let $f$ be a holomorphic function on $\Omega$, and let $E \subseteq \partial \Omega$ be a set of positive $\sigma$ measure. If $f$ has admissible boundary limit 0 at each point of $E$ then $f \equiv 0$.

Proof: Near a point of $\sigma$-density of $E$, we may find a parametrized family of transversal complex lines $\ell$, with the parametrizing set being real-twodimensional and so that (i) each $\ell \cap \partial \Omega$ is a smooth curve and (ii) each $\ell \cap E$ is a subset of $\ell \cap \partial \Omega$ having positive linear measure. For each such $\ell,\left.f\right|_{\ell \cap \Omega}$ is a holomorphic function of one complex variable that has nontangential boundary limit 0 on a set of positive linear measure. So $\left.f\right|_{\ell \cap \Omega} \equiv 0$. Since this conclusion is true on each $\ell$, we conclude that $f \equiv 0$ on $\Omega$.

\section{Concluding Remarks}

The purpose of this paper has been to supply proofs of results that come up frequently in function-theoretic arguments of several complex variables, but which are not well recorded in the literature. We hope that the arguments and results presented here will prove to be useful in other contexts. Certainly the content of this paper nicely complements the ideas in [KRA1, Ch. 8] and [STE1]. 


\section{References}

[BAS] F. Bagemihl and W. Seidel, Some boundary properties of analytic functions, Math. Zeitschr. 61(1954), 186-199.

[BEK] S. R. Bell and S. G. Krantz, Smoothness to the boundary of conformal mappings, Rocky Mountain J. Math. 17(1987), 23-40.

[BUR] R. Burckel, An Introduction to Classical Complex Analysis, Vol. 1, Pure and Applied Mathematics, 82, Academic Press, Inc., New YorkLondon, 1979.

[CIK] J. A. Cima and S. G. Krantz, A Lindelöf principle and normal functions in several complex variables, Duke Math. Jour. 50(1983), 303-328.

[COL] E. F. Collingwood and A. J. Lohwater, The Theory of Cluster Sets, Cambridge University Press, Cambridge, 1966.

[DUR] P. Duren, The Theory of $H^{p}$ Spaces, Academic Press, New York, 1970.

[FEF] C. Fefferman, The Bergman kernel and biholomorphic mappings of pseudoconvex domains, Invent. Math. 26(1974), 1-65.

[GRK] R. E. Greene and S. G. Krantz, Function Theory of One Complex Variable, 3rd ed., American Mathematical Society, Providence, RI, 2006.

[HAS] M. Hakim and N. Sibony, Boundary properties of holomorphic functions in the ball of $\mathbb{C}^{n}$, Math. Ann. 276(1987), 549-555.

[HIR] M. Hirsch, Differential Topology, Springer-Verlag, New York, 1976.

[KRA1] S. G. Krantz, Function Theory of Several Complex Variables, 2nd ed., American Mathematical Society, Providence, RI, 2001.

[KRA2] S. G. Krantz, Partial Differential Equations and Complex Analysis, CRC Press, Boca Raton, FL, 1992.

[KRA3] S. G. Krantz, The Lindelöf principle in several complex variables, Journal of Math. Anal. and Applications 326(2007), 1190-1198. 
[LEV] O. Lehto and K. Virtanen, Boundary behavior and normal meromorphic functions, Acta Math. 97(1957), 47-65.

[LEM] L. Lempert, Boundary behavoiur of meromorphic functions of several variables, Acta Mathematica 144(1980), 1-25.

[LUM] G. Lumer, Espaces de Hardy en plusiers variables complexes, C. R. Acad. Sci. Paris, Sér. A-B 273(1971), 151-154.

[NAR] A. Nagel and W. Rudin, Local boundary behavior of bounded holomorphic functions, Canadian Journal of Mathematics 30(1978), 583-592.

[POM] C. Pommerenke, Boundary Behaviour of Conformal Maps, Grundlehren der Mathematischen Wissenschaften, 299, Springer-Verlag, Berlin, 1992.

[RUD] W. Rudin, Lumer's Hardy spaces, Mich. Jour. Math. 24(1977), 1-5.

[STE1] E. M. Stein, Boundary Behavior of Holomorphic Functions of Several Complex Variables, Princeton University Press, Princeton, NJ, 1972.

[STE2] E. M. Stein, Singular Integrals and Differentiability Properties of Functions, Princeton University Press, Princeton, NJ, 1970.

Steven G. Krantz

Department of Mathematics

Washington University in St. Louis

St. Louis, Missouri 63130

sk@math. wustl . edu 